CAHIERS DE

NARRATOLOGIE

\section{Cahiers de Narratologie}

Analyse et théorie narratives

$26 \mid 2014$

Nouvelles frontières du récit. Au-delà de l'opposition entre factuel et fictionnel

\title{
Compte rendu : «Événement et roman. Une relation critique »
}

Événement et roman. Une relation critique, Marc Courtieu, AmsterdamNew York, Rodopi, 2012, 337 p.

\section{Anne-Laure Bonvalot}

\section{(2) OpenEdition}

\section{Journals}

Electronic version

URL: http://journals.openedition.org/narratologie/6903

DOI: $10.4000 /$ narratologie.6903

ISSN: $1765-307 X$

Publisher

LIRCES

Electronic reference

Anne-Laure Bonvalot, «Compte rendu : «Événement et roman. Une relation critique » », Cahiers de Narratologie [Online], 26 | 2014, Online since 20 September 2014, connection on 22 September 2020. URL : http://journals.openedition.org/narratologie/6903; DOI : https://doi.org/10.4000/narratologie. 6903

This text was automatically generated on 22 September 2020

Article L.111-1 du Code de la propriété intellectuelle. 


\section{Compte rendu : «Événement et roman. Une relation critique »}

Événement et roman. Une relation critique, Marc Courtieu, AmsterdamNew York, Rodopi, 2012, 337 p.

\section{Anne-Laure Bonvalot}

1 Cet ouvrage est consacré à l'étude du passage d'un modèle de mise en récit romanesque de l'événement à un autre, et propose d'éclairer les enjeux dans le roman de la coupure épistémologique survenue à la fin du XIXe siècle. Au sein de ce procès, il s'agit de mesurer les ruptures et les persistances qui marquent ce qui est défini comme un véritable changement de paradigme. De "l'événementiel classique » au " nouvel événemential» (p. 11), cette nouvelle vision du monde qui se fait jour aux alentours de 1860, on assiste à une reconfiguration radicale du statut et de la nature de l'événement - mutation que l'auteur choisit d'appréhender au moyen d'un parcours à travers une multitude de formes et de figures romanesques de l'événement. Celui-ci ne se limite plus à advenir aux personnages, il dépasse sa seule existence " intramondaine " pour acquérir ses autres dimensions constitutives, " discursive " et "opérale ", pour reprendre avec Marc Courtieu la terminologie de Claude Romano et de René Audet, présente en filigrane dans l'entier du raisonnement.

2 L'ouvrage se compose de six parties d'inégale longueur, précédées d'une introduction synthétique et d'une conclusion efficace, architecture que vient compléter une bibliographie substantielle de vingt-huit pages.

3 La première partie, composée de quatre chapitres, dresse un état des lieux du traitement de l'événement dans le grand roman réaliste du XIXe siècle. Marc Courtieu s'attache à examiner ce qu'il pose comme étant le statut classique de l'événementiel. Il en égrène les caractéristiques spécifiques, et souligne le rôle moteur, central et nécessaire de l'événement dans le récit, en particulier balzacien. Croisant en permanence science biologique et écriture romanesque, le propos met en lumière les liens unissant les récits de Cuvier et ceux d'un Balzac : c'est à l'aune de la catastrophe, du surgissement de la discontinuité ou encore du saut irréductible, que l'événement est alors envisagé. Ce «paradigme essentialiste » (p. 53), tributaire de la théorie 
créationniste largement dominante, fait de l'événement le cœur d'une conception fondamentalement téléologique $\mathrm{du}$ romanesque, son traitement induisant nécessairement une organisation narrative orientée vers une fin. L'événement revêt alors un statut profondément paradoxal, puisque l'altération dont il est porteur a en dernière instance pour effet de confirmer aussi bien la continuité du récit que « le monde en sa bonne marche » (p. 297).

Cet étalon établi - événement intramondain, temporel, central et paradoxal -, la deuxième partie, organisée en deux chapitres, propose une analyse de la progressive mise en doute du paradigme initial - dans les romans de Melville, puis dans ceux de Flaubert ou des naturalistes -, après la rupture fondamentale que constitue dans la représentation de la temporalité la révolution darwinienne. L'avènement d'une nouvelle vision du monde est aussi celui d'une nouvelle manière de mise en récit, et l'auteur d'envisager les effets et les influences dans l'ordre du roman de ces nouveaux modèles de scientificité que sont l'évolutionnisme et le continuisme darwiniens. Il est notamment question des inflexions majeures que le réalisme flaubertien et le naturalisme font subir au concept d'événement. Alors que la question de l'essence langagière de ce dernier apparaît pour la première fois sous la plume de Flaubert, chez les naturalistes, la prégnance du déterminisme et le principe de continuité sur lequel il repose se traduisent dans l'ordre narratif par une réduction ou un déplacement du rôle de l'événement, qui quitte les oripeaux de l'inattendu et sort de sa seule fonction actantielle pour réinvestir le récit romanesque sous d'autres formes, que l'auteur inventorie avec finesse. Toutefois, malgré ces modifications substantielles, l'événement reste encore ce « mal nécessaire » (p. 78) sous les auspices duquel l'agencement du récit romanesque continue d'être pensé.

5 La troisième partie, distribuée en cinq chapitres, envisage la tension entre continu et discontinu au prisme d'une dialectique de l'aventure et du quotidien, dialectique s'actualisant notamment dans la fiction sous la forme particulière du roman d'aventures, marqué par une hypertrophie de l'événementiel, par une recherche compulsive de la catastrophe. C'est en partie contre l'insupportable monotonie de l'ennuyeux récit naturaliste que la prose d'un Stevenson ou d'un London s'érige, réaction qui se traduit par une démultiplication de l'événement " aventureux » (p. 79) frappant, héroïque, exacerbé, primitif. Ce retour en force de l'événement est tel qu'il a pu faire - si l'on en croit une certaine vulgate contre laquelle Marc Courtieu s'inscrit en faux - de l'événement aventureux l'essence de la fiction romanesque. Le tour plus monographique que prend ici le propos permet à l'auteur d'opposer cette rhétorique de l'événement frénétique à une écriture des représentations dégradées de l'aventure, ramollie par les puissances dissolvantes de l'ennui (Céline), à des poétiques de la durée, du « ratage " (p. 105) de l'aventure (Sartre, Chevillard, Le Clézio), ou encore à des écritures de l'entre-deux (Conrad, James).

Dans la même logique, la quatrième partie, organisée en quatre chapitres, s'attarde sur la naissance - dès Melville - d'une nouvelle rhétorique événementielle dans le roman nord-américain, explorant notamment la notion au travers des liens indissolubles qui l'unissent au concept fondateur de la "Fronteer ». Une spatialisation ou une territorialisation de la pensée de l'événement est à l'œuvre, qui induit des modes de saisie radicalement nouveaux. Si la frontière en mouvement est le lieu privilégié de condensation de l'imaginaire de l'événement, alors la fiction se fait plus rapide, plus mobile, plus immédiate : c'est du déplacement que naissent les événements, et l'auteur 
d'évoquer une véritable « idéologie de l'aventure » (p. 139) propre à un roman nordaméricain qui sans cesse rejoue à travers elle la partition de la frontière en tant que mythe fondateur. Un intermède vient clore ce quatrième moment, qui propose un portrait de l'écrivain en infatigable copiste, au travers des figures emblématiques de Bartleby (Melville) et de Bouvard et Pécuchet (Flaubert) : l'événement classique, inattendu et nouveau, vacille définitivement, et la problématique de la copie sans original pose avec acuité la question de l'essence - intramondaine ou discursive ? - de l'événement. D'aucuns ont vu dans ces chancellements une mise à mort de l'événement par et dans le roman. Pourtant, la mise en récit de l'impossible rencontre entre l'événement et le langage de représentation, ou du doute radical sur la capacité du langage à dire le monde, contribueront à renforcer l'aura de l'événement, qui devient cet introuvable autour duquel le récit tourne inlassablement. Est-ce à dire que l'écriture en tant que " pratique gestuelle » (Barthes, p. 197) est le seul événement qui subsiste, celui-ci n'existant plus au-delà du récit qui le prend en charge ?

7 Pour répondre à cette interrogation, la cinquième partie, divisée en cinq chapitres qui sont autant d'études de cas, ainsi que le sixième et dernier moment consacré à Beckett, proposent un parcours plus délibérément monographique au travers des devenirs de l'événement " après le changement ». Ce n'est pas par l'hypertrophie de l'aventure toujours recommencée que le roman d'« un certain XXe siècle » (p. 12) répond à la question philosophique de l'articulation du discours au monde, mais par un processus d'étiolement ou de glissement de l'événement. De Joyce à Sarraute en passant par Woolf, Broch ou Musil, on n'a pas affaire à une évacuation pure et simple de l'objet, mais plutôt à l'entreprise - diffractée en une multitude de figures que le propos commente minutieusement - de sa reconfiguration essentielle : de pure extériorité qui advient, l'événement migre vers l'intérieur ; il ne sert plus à asseoir la réalité du monde ou à en confirmer par son surgissement même le cours tranquille, mais devient cet élément central dont seule l'advenue permet à la réalité d'exister ; il est cette synthèse ontologique, ce " moment d'être » (Woolf), cet " instant d'Unicité » (Broch), où se rencontrent le lointain intérieur et la radicale extériorité du monde. Au rebours de la conception catastrophiste, l'événement devient interface, il est une figure rare, intermittente, épiphanique, de l'abolition de toutes les frontières - entre le sujet et l'objet, le dedans et le dehors, la connaissance scientifique et la connaissance intuitive (Musil), le mot et la chose, le temps et l'éternité. Il est tantôt stase ou atome de l'histoire, tantôt ouverture ; il est toujours ce moment d'indifférenciation que l'écriture traque et cherche à provoquer.

8 L'étude finale fait une place à part aux « romans » de Samuel Beckett. Malgré le travail "d'abstraction-intraction » (p. 278) auquel ce dernier se livre, malgré ce minimalisme qui a rendu possible une lecture purement formaliste de son œuvre, le propos éclaire avec brio l'attention inégalée que portait l'auteur à l'événement pur, ce "grain de sable de l'histoire »(p. 288), qui se tient là, malgré tout, irréductible. S'évertuant à décortiquer, à épurer, à désintégrer, ni Sarraute ni Beckett n'ont réellement évacué l'événement du récit, tout au contraire. S'opposant aux théories qui voient dans ces écritures une mise au ban ou un dépassement de l'événement, Marc Courtieu montre que la mise en cause des déterminations, des logiques narratives explicatives ou des évidences de la représentation a plutôt fait du langage l'événement de son propre dire. L'événement, atomisé, grandiose ou minuscule, rendu à sa pureté minimale et 
désormais ressaisi sous les auspices du commencement, du rythme, ou du moment originel que l'écriture actualise sans fin, demeure au roman proprement essentiel.

9 Cette étude magistrale n'aurait pu avoir qu'un tort, que l'auteur prévient et dissipe dans la conclusion, c'est précisément de faire du " roman classique » un idéal, une pure origine qui contiendrait l'événement canonique, plein et entier, et à partir duquel l'histoire du concept prendrait la forme d'une lente et irrémédiable dégradation. Mais Marc Courtieu évite ce double écueil - essentialiste et téléologique - en mettant en lumière le caractère constructiviste et stratégique de sa démarche : le " roman classique » n'est qu'une catégorie heuristique, une « tradition inventée [...], construite pour mieux s'y opposer » (p. 305), répondant à des fins purement pratiques. Gageons que cette contribution - sa qualité théorique, l'intelligence de ses analyses, la beauté de ses développements - saura créer l'événement dans le champ des études narratologiques.

\section{AUTHOR}

\section{ANNE-LAURE BONVALOT}

Université Paul Valéry - Montpellier III ; Équipe de recherche: LLACS 